\title{
The spectrum of response to erenumab in patients with chronic migraine and subgroup analysis of patients achieving $\geq \mathbf{5 0} \%, \geq \mathbf{7 5} \%$, and $100 \%$ response
}

Cephalalgia

2020, Vol. 40(I) 28-38

(C) International Headache Society 2019 Article reuse guidelines: sagepub.com/journals-permissions DOI: I0.1I77/03331024I9894559 journals.sagepub.com/home/cep

@SAGE

\author{
Jan Lewis Brandes', Hans-Christoph Diener², David Dolezil', \\ Marshall C Freeman, ${ }^{4}$ Peter J McAllister ${ }^{5}$, Paul Winner 6 , \\ Jan Klatt ${ }^{7}$, Sunfa Cheng ${ }^{8}$, Feng Zhang ${ }^{8}$, Shihua Wen?, \\ Shannon Ritter ${ }^{9}$, Robert A Lenz ${ }^{8}$ and Daniel D Mikol ${ }^{8}$
}

\begin{abstract}
Objective: To assess the efficacy of erenumab across the spectrum of response thresholds ( $\geq 50 \%, \geq 75 \%, 100 \%)$ based on monthly migraine days (MMD) reduction in patients with chronic migraine from a 12 -week, randomized study (NCT020664l5).

Methods: Patients $(n=667)$ received (3:2:2) placebo or erenumab 70/l40 mg once-monthly. The proportion of patients achieving a given response threshold was assessed. A post-hoc analysis was conducted to contextualize the actual treatment benefit in subgroups of patients achieving (or not) specified response thresholds. Outcome measures included MMD, acute migraine-specific medication treatment days (MSMD) and disability.

Results: The proportion of patients responding to erenumab exceeded that of placebo at the $\geq 50 \%$ and $\geq 75 \%$ response thresholds. At month 3, 39.9\% and 4I.2\% of patients on erenumab 70 and $140 \mathrm{mg}$, respectively, achieved $\geq 50 \%$ response versus placebo (23.5\%). Similarly, at month $3,17.0 \%$ and $20.9 \%$ of patients on erenumab 70 and $140 \mathrm{mg}$, respectively, achieved $\geq 75 \%$ response versus placebo (7.8\%). Compared with the overall erenumab-treated population (change in MMD: -6.6 [both 70 and $140 \mathrm{mg}$ ]), $\geq 50 \%$ responders showed MMD reductions of $-12.2 /-12.5$ for $70 \mathrm{mg} / 140 \mathrm{mg}$ versus $-2.6 /-2.2$ for those not achieving $\geq 50 \%$ response. $\geq 75 \%$ responders showed $M M D$ reductions of $-13.9 /-14.8$ for $70 \mathrm{mg} / \mathrm{l} 40 \mathrm{mg}$ versus $-5.0 /-4.3$ for those not achieving $\geq 75 \%$ response. Relative improvements in MSMD and disability were observed in responders versus overall erenumab-treated population.

Conclusion: For erenumab-treated patients achieving $\geq 50 \%$ response, the actual reduction in MMD was almost twice that of the overall population. These findings provide context for setting realistic expectations regarding actual treatment benefit experienced by patients responding to treatment.
\end{abstract}

\section{Keywords}

Erenumab, chronic migraine, monthly migraine days, responder rates

Date received: 7 October 2019; revised: I8 November 2019; accepted: 2I November 2019

\footnotetext{
'Nashville Neuroscience Group, NUMC, Vanderbilt University, Department of Neurology, Nashville, TN, USA

${ }^{2}$ Faculty of Medicine, University Duisburg-Essen, Essen, Germany

${ }^{3}$ Prague Headache Center, DADO MEDICAL s.r.o., Prague, Czech Republic

${ }^{4}$ Headache Wellness Center, Greensboro, NC, USA

${ }^{5} \mathrm{New}$ England Institute for Neurology and Headache, Stamford, CT, USA
}

\footnotetext{
${ }^{6}$ Palm Beach Headache Center, West Palm Beach, FL, USA

${ }^{7}$ Novartis Pharma AG, Basel, Switzerland

${ }^{8}$ Amgen Inc., Thousand Oaks, CA, USA

${ }^{9}$ Novartis Pharmaceuticals Corporation, East Hanover, NJ, USA

Corresponding author:

Jan Lewis Brandes, Nashville Neuroscience Group, NUMC, Vanderbilt University, Department of Neurology, Nashville, TN, USA.

Email: jbrandes I@msn.com
} 


\section{Introduction}

Chronic migraine (CM) affects approximately $1-2 \%$ of the global population (1). According to the International Classification of Headache Disorders (ICHD-III) criteria, CM is defined as headache occurring on 15 or more days per month for more than 3 months, which has the features of migraine headache on at least 8 days per month (2). The high frequency of migraine and non-migraine headache days has a substantial impact on the socioeconomic status of patients, their role functioning and on their quality of life (QoL) (3). Patients with CM have more healthcare provider and emergency visits than those with episodic migraine (4). Due to the high frequency of headaches and severe impact on QoL, patients with CM are generally prescribed preventive medications. However, there is a considerable inter-patient variability in the tolerability and efficacy of the available preventive treatment options $(5,6)$.

Currently available oral migraine preventive medications are associated with low adherence rates due to lack of efficacy and/or poor tolerability (6-8). In a retrospective claims analysis of 8707 patients in the USA, poor persistence $(25 \%)$ was observed after the first 6 months of treatment, and it further declined to $14 \%$ by 12 months (9). In clinical practice, patients who tolerate and achieve a clinically meaningful response to treatment are likely to continue treatment, whereas those not achieving an adequate response or who experience adverse events are likely to discontinue treatment. Therefore, it is clinically relevant to understand the actual degree of benefit in patients achieving certain thresholds of response because those achieving benefit are more likely to remain on treatment.

Erenumab is a fully human monoclonal antibody that selectively targets and blocks the canonical calcitonin gene-related peptide receptor (10). It is approved as a preventive treatment for adults with migraine in the US and the EU $(11,12)$. Patients with CM treated with erenumab (70 and $140 \mathrm{mg}$ ) demonstrated significant reductions in the primary endpoint of monthly migraine days (MMD) in a 12-week, pivotal, placebocontrolled study (NCT02066415) (baseline: 18.0 days; reduction of 6.6 days for both 70 and $140 \mathrm{mg}$ versus 4.2 days for placebo, [both $p<0.001]$ ). Compared with placebo, a greater proportion of patients treated with erenumab 70 and $140 \mathrm{mg}$ achieved $\geq 50 \%$ reduction in MMD from baseline (secondary endpoint) (13), which is considered a clinically meaningful response $(14,15)$.

Here, we report response rates at the $\geq 50 \%, \geq 75 \%$ and $100 \%$ thresholds (as defined by relative reduction from baseline in MMD) as well as nonresponse $(\leq 0 \%)$ from this study (13) and contextualize the actual benefit achieved in the subgroups of patients responding at the above thresholds.

\section{Methods}

\section{Patient eligibility criteria}

Patient eligibility criteria have been described previously (13). Briefly, men and women aged 18-65 years with a history of CM ( $\geq 15$ headache days per month, of which $\geq 8$ were migraine days; with or without aura) were included in the study. Patients with overuse of triptans, ergot derivatives, simple analgesics, and combinations of drugs (any combination of those above, combination analgesics, or opiates) were permitted to participate in the study (13).

Patients aged $\geq 50$ years at migraine onset and a history of cluster headache or hemiplegic migraine, or $\mathrm{CM}$ with continuous pain (i.e. no pain-free periods of any duration during the 1 month before screening) were excluded. Patients with no therapeutic response to more than three categories of preventive treatment were also excluded.

\section{Study design}

This was an exploratory analysis of data from a multicenter, randomized, double-blind placebo-controlled study (NCT02066415) of patients with CM conducted in 69 headache and clinical research centres in North America (Canada and the USA) and Europe (Czech Republic, Denmark, Finland, Germany, Norway, Poland, Sweden, and the UK). The study comprised an initial screening phase (up to 3 weeks), baseline (4 weeks), a double-blind treatment phase (DBTP; 12 weeks), and a safety follow-up phase (12 weeks) (13). Eligible patients were randomly assigned (3:2:2) to receive placebo, erenumab $70 \mathrm{mg}$, and erenumab $140 \mathrm{mg}$, once every 4 weeks for the 12-week DBTP (13).

An independent ethics committee or relevant institutional review board for each study center approved the final study protocol. The study was conducted in accordance with International Conference on Harmonization Good Clinical Practice regulations/ guidelines, and in accordance with the ethical principles set forth in the Declaration of Helsinki. All centers complied with local regulations. All patients provided written informed consent before study initiation. Summaries of data by unmasked treatment assignment were prepared by an independent biostatistician group and reviewed by an independent data monitoring committee.

\section{Endpoints and assessments}

Results for the primary endpoint, change from baseline in MMD to the last 4 weeks (month 3 ) of the 12-week DBTP, have been reported previously (13). The responder rates were derived from percentage change from 
baseline in MMD at each time point; that is, to month 1 , month 2 or month 3 (primary time point).

Waterfall plot analysis of change in MMD from baseline. Waterfall plots were generated to show the change in MMD from baseline to Month 3 for individual patients in each treatment group (bars above baseline indicate worsening and bars below baseline indicate reduction in MMD [improvement]).

Responder rate analyses. The responder rate analyses included achievement of $\geq 50 \%$ (pre-specified secondary endpoint), $\geq 75 \%$ (post hoc analysis), and $100 \%$ (post hoc analysis) reduction in MMD from baseline to Month 3. In addition, the percentage of patients with no response to treatment, defined as no change or worsening of MMD, was assessed (post hoc analysis).

Efficacy and patient-reported outcomes in subgroups based on various thresholds of MMD response. A post hoc analysis was also conducted to contextualize the actual treatment benefit among patients achieving or not achieving response at the $\geq 50 \%, \geq 75 \%$, and $100 \%$ thresholds. Outcome measures assessed were change from baseline to month 3 in MMD, migraine-specific medication treatment days (MSMD), the Headache Impact Test (HIT-6) scores, and Migraine Disability Assessment (MIDAS) total scores.

Statistical analysis. The proportions of patients with $\geq 50 \%, \geq 75 \%$, and $100 \%$ reduction in MMD were calculated from baseline to month 3 . Odds ratios (ORs) and associated $p$-values for each erenumab dose versus placebo were obtained from the Cochran-Mantel-Haenszel test using data including placebo and the corresponding erenumab dose group alone. Missing data was imputed using non-responder imputation (NRI). The efficacy analyses set included patients who received either placebo, erenumab $70 \mathrm{mg}$, or $140 \mathrm{mg}$ and had at least one postbaseline monthly eDiary measurement. For $\geq 50 \%$ reduction from baseline in MMD, statistical significance was determined based on a pre-specified hierarchical gatekeeping procedure adjusted for multiplicity. Statistical significance for post hoc endpoints of $\geq 75 \%$ and $100 \%$ reduction in MMD were determined by comparing nominal $p$-values with a significance level of 0.05 . Analysis of efficacy in responder subgroups at the $\geq 50 \%, \geq 75 \%$, and $100 \%$ thresholds was based on descriptive statistics alone.

\section{Results}

\section{Patient disposition and baseline characteristics}

Patient disposition and baseline characteristics have been reported previously (13). In summary, a total of
667 patients were randomized. The mean age of the patients was $42.1,41.4$, and 42.9 years in the placebo, erenumab $70 \mathrm{mg}$, and $140 \mathrm{mg}$ treatment groups, respectively. The majority of patients were women (placebo: $79.0 \%$, erenumab $70 \mathrm{mg}: 86.9 \%$ and erenumab $140 \mathrm{mg}: 84.2 \%$ ). The mean (standard deviation [SD]) MMD at baseline was 18.2 (4.7), 17.9 (4.4), and 17.8 (4.7) days in the placebo, erenumab $70 \mathrm{mg}$, and $140 \mathrm{mg}$ groups, respectively. The mean (SD) monthly acute migraine-specific medication use at baseline was 9.5 (7.6), 8.8 (7.2), and 9.7 (7.0) days in the placebo, erenumab $70 \mathrm{mg}$, and $140 \mathrm{mg}$ groups, respectively. The proportion of patients who failed $\geq 1$ prior preventive treatment due to lack of efficacy or poor tolerability were $69.9 \%, 66.5 \%$, and $66.3 \%$ in the placebo, erenumab $70 \mathrm{mg}$, and $140 \mathrm{mg}$ groups, respectively.

Waterfall plot analysis of change in MMD from baseline. In the overall DBTP population, a higher number of patients showed reduction in MMD from baseline in the erenumab 70 and $140 \mathrm{mg}$ groups versus placebo (patients showing reduction in MMD: placebo, $\mathrm{n}=191$; erenumab $70 \mathrm{mg}, \mathrm{n}=149$; erenumab $140 \mathrm{mg}, \mathrm{n}=144$; patients with no change or worsening: placebo, 66; erenumab $70 \mathrm{mg}$, $\mathrm{n}=26$; erenumab $140 \mathrm{mg}, \mathrm{n}=35$; Figure 1).

Spectrum of response. As presented in Figure 2, regardless of threshold used for the responder definition, a higher percentage of erenumab-treated patients achieved responses compared with patients receiving placebo. The proportion of patients achieving $\geq 50 \%$, $\geq 75 \%$, and $100 \%$ response is presented in Figure 2 . Overall, $28.1 \%, 16.3 \%$, and $20.9 \%$ of patients demonstrated no change or worsening in the placebo, erenumab $70 \mathrm{mg}$, and $140 \mathrm{mg}$ groups, respectively.

$\geq 50 \%$ responder rate. At month 3,39.9\% $(\mathrm{n}=75 / 188)$ and $41.2 \%(\mathrm{n}=77 / 187)$ of patients treated with erenumab $70 \mathrm{mg}$ and $140 \mathrm{mg}$, respectively, achieved $\geq 50 \%$ reduction from baseline in MMD compared with $23.5 \%(n=66 / 281)$ treated with placebo. The odds of achieving $\geq 50 \%$ reduction in MMD from baseline to month 3 were significantly greater in patients treated with erenumab compared with placebo (erenumab $70 \mathrm{mg}$ : OR 2.2, 95\% confidence interval [CI]: 1.5-3.3, $p<0.001$; erenumab $140 \mathrm{mg}$ : OR $2.3,95 \% \mathrm{CI}: 1.6-3.5$, $p<0.001$; Figure 3) and this effect was observed from month 1 (week 4) onwards. The proportion of patients who achieved $\geq 50 \%$ response in one or more of the 3 monthly treatment periods in the placebo, erenumab $70 \mathrm{mg}$, and $140 \mathrm{mg}$ groups were $30.6 \%, 57.4 \%$, and $54.0 \%$, respectively.

$\geq 75 \%$ responder rate. At month 3, 17.0\% $(n=32 / 188)$ and $20.9 \% \quad(\mathrm{n}=39 / 187)$ of patients treated with 


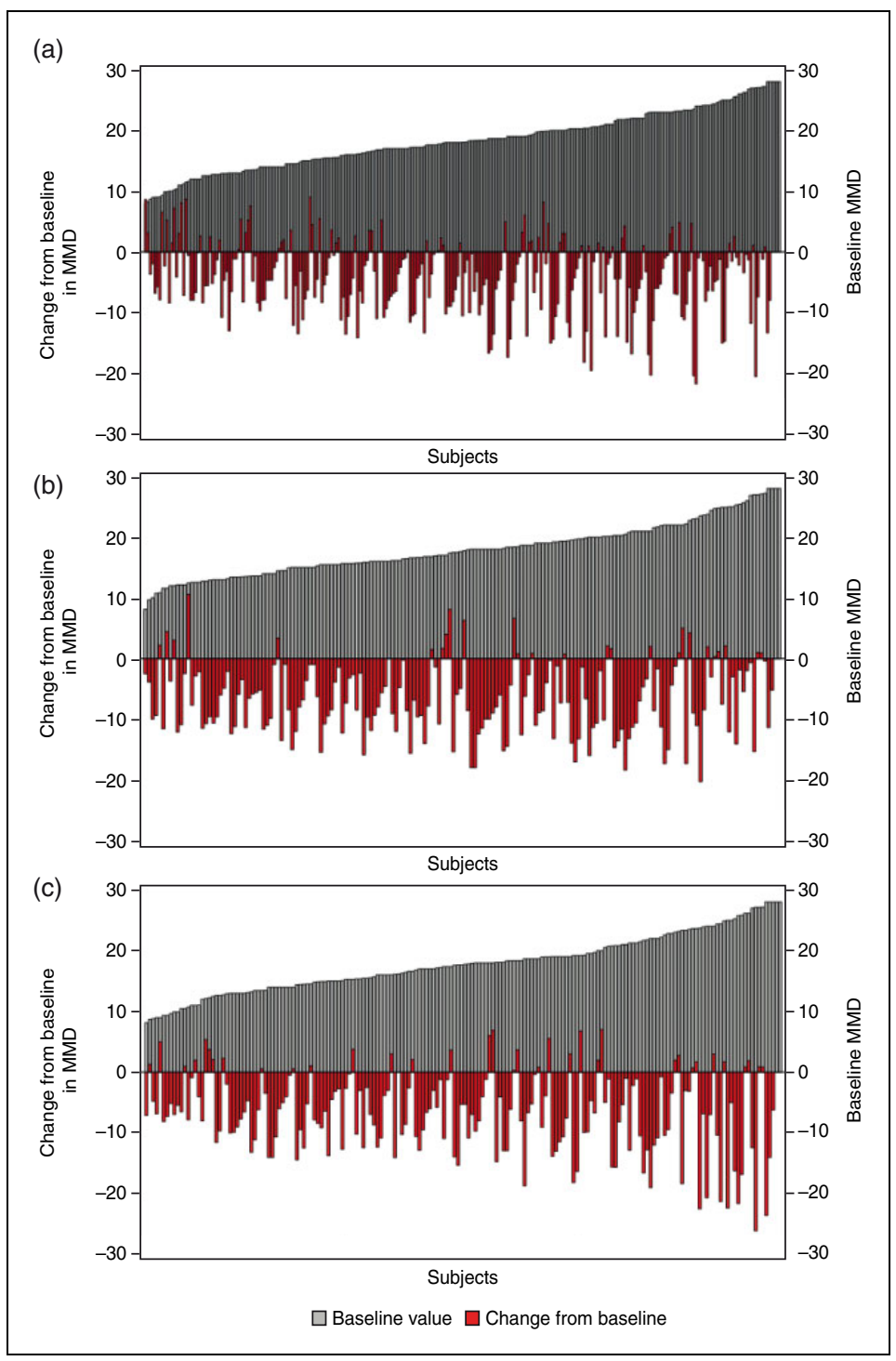

Figure I. Waterfall plot of change in MMD from baseline versus baseline MMD (efficacy analysis set), (a) placebo (b) erenumab $70 \mathrm{mg}$ (c) erenumab $140 \mathrm{mg}$.

Bars above baseline indicate worsening and bars below baseline indicate reduction in MMD [improvement]). MMD: monthly migraine days.

erenumab $70 \mathrm{mg}$ and $140 \mathrm{mg}$, respectively, achieved $\geq 75 \%$ reduction from baseline in MMD compared with $7.8 \%(n=22 / 281)$ of patients treated with placebo. The odds of achieving $\geq 75 \%$ reduction in MMD from baseline to month 3 were significantly greater with erenumab 70 and $140 \mathrm{mg}$ compared with placebo (erenumab $70 \mathrm{mg}$ : OR $2.4,95 \%$ CI: $1.4-4.3, p=0.002$; erenumab $140 \mathrm{mg}$ : OR $3.1, \quad 95 \%$ CI: $1.8-5.5$, $p<0.001$; Figure 4). The proportion of patients who achieved $\geq 75 \%$ response in one or more of the 3 monthly treatment periods in the placebo, erenumab $70 \mathrm{mg}$, and $140 \mathrm{mg}$ groups were $12.8 \%, 25.0 \%$, and $27.3 \%$, respectively.
$100 \%$ responder rate. Achievement of $100 \%$ reduction from baseline in MMD is a high bar for a CM population. The proportion of patients who achieved a $100 \%$ response at month 3 in the placebo, erenumab $70 \mathrm{mg}$, and $140 \mathrm{mg}$ groups were $0.4 \% \quad(\mathrm{n}=1 / 281), 4.3 \%$ $(\mathrm{n}=8 / 188)$, and $2.7 \%(\mathrm{n}=5 / 187)$, respectively.

Efficacy and patient-reported outcomes in subgroups based on response at various thresholds. Among the $\geq 50 \%$ responders, the baseline MMD was 17.1 and 17.0 for erenu$\mathrm{mab} 70 \mathrm{mg}$ and erenumab $140 \mathrm{mg}$, respectively (compared with 18.0 and 17.8 for the overall erenumab $70 \mathrm{mg}$ and erenumab $140 \mathrm{mg}$ groups, respectively; 


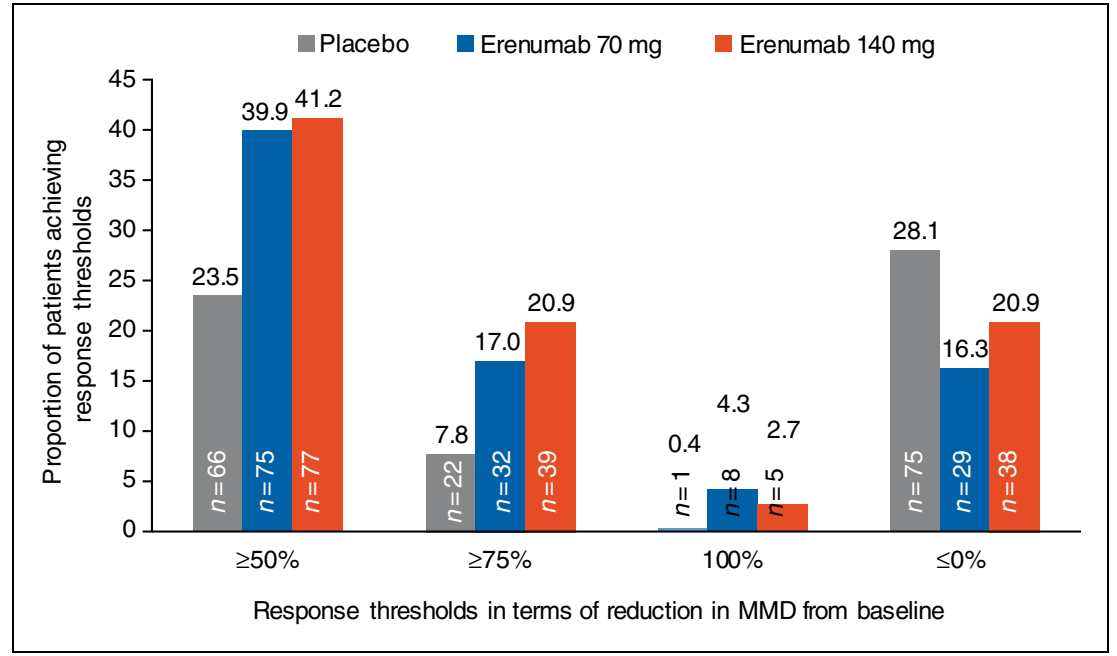

Figure 2. Distribution of response rate, based on relative change from baseline in MMD at month 3. MMD, monthly migraine days.

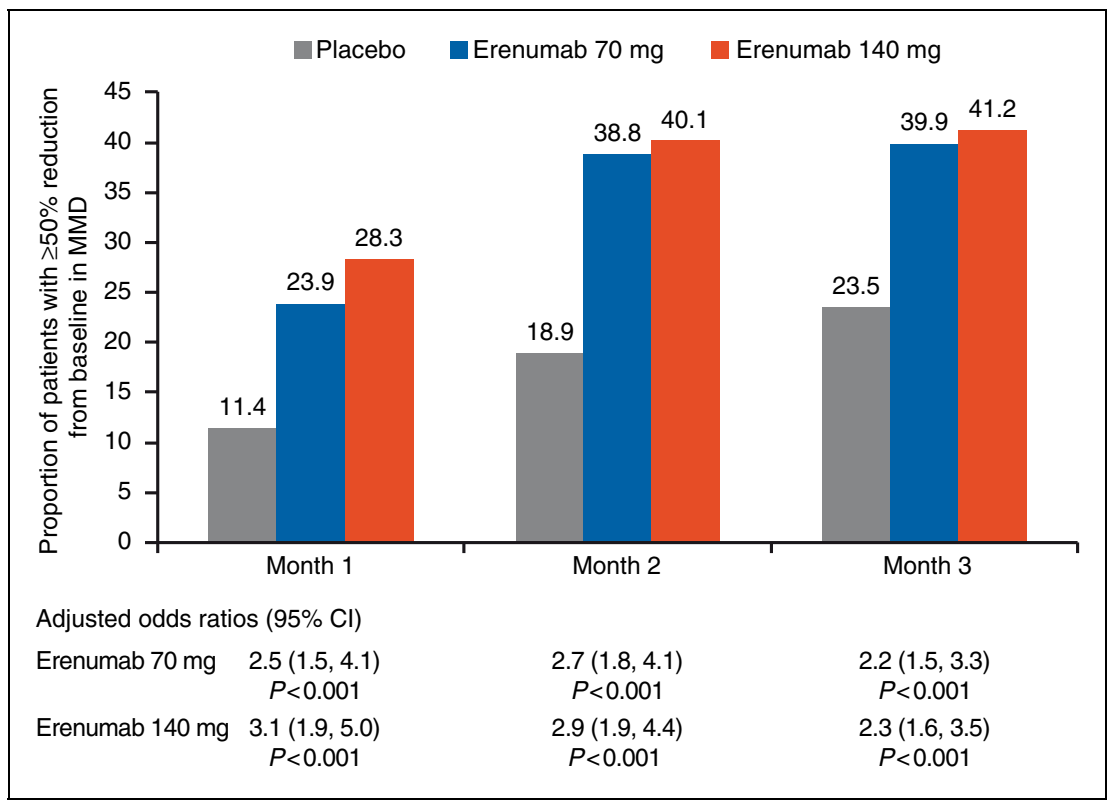

Figure 3. Proportion of patients with $a \geq 50 \%$ reduction from baseline in MMD.

$\mathrm{N}$ : Placebo, 28I; Erenumab $70 \mathrm{mg}, 188$; Erenumab $140 \mathrm{mg}, 187$.

The adjusted odds ratios and $p$-values were obtained from a Cochran-Mantel-Haenszel $(\mathrm{CMH})$ test after the missing data are imputed as non-response, stratified by stratification factors region and medication overuse. The same analysis is repeated for each visit. $p$-values for pairwise comparisons are nominal $p$-values obtained from the $\mathrm{CMH}$ test using data including placebo and the corresponding erenumab dose group only.

$\mathrm{Cl}$ : confidence interval; MMD: monthly migraine days; $\mathrm{N}$ : total number of patients.

Table 1). For the subgroup of patients with response at a $\geq 50 \%$ threshold, mean reductions from baseline of $-12.2(70 \mathrm{mg})$ and $-12.5(140 \mathrm{mg})$ MMD were achieved (Figure 5).

Greater reductions in MSMD, HIT-6, and MIDAS total scores were observed for both doses of erenumab in the responder subgroups compared with the overall erenumab-treated population (Figures 6-8). For the subgroup of patients with a response at the $\geq 75 \%$ and $100 \%$ thresholds an even greater reduction in MMD was observed than in the overall erenumab-treated population (Figure 5). The change in MSMD was 


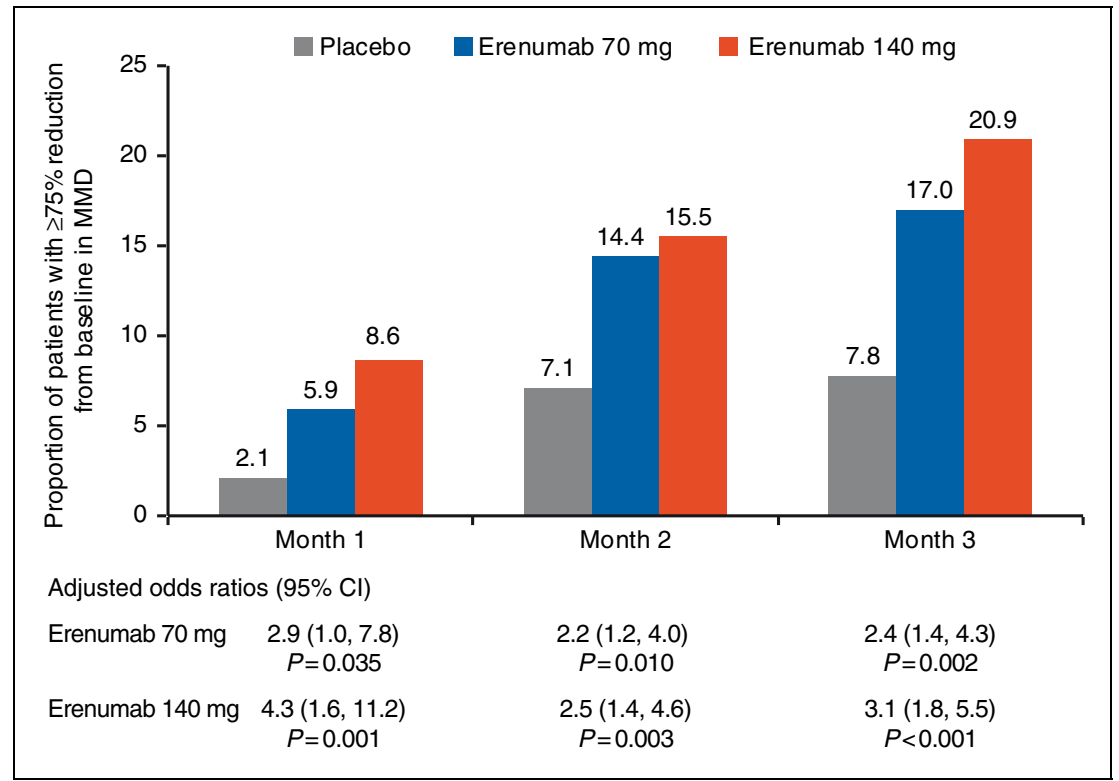

Figure 4. Proportion of patients with a $\geq 75 \%$ reduction from baseline in MMD.

$\mathrm{N}$ : Placebo, 28I; Erenumab $70 \mathrm{mg}, 188$; Erenumab $140 \mathrm{mg}, 187$.

The adjusted odds ratios and $p$-values were obtained from a Cochran-Mantel-Haenszel $(\mathrm{CMH})$ test after the missing data are imputed as non-response, stratified by stratification factors region and medication overuse. The same analysis is repeated for each visit. $p$-values for pairwise comparisons are nominal $p$-values obtained from the $\mathrm{CMH}$ test using data including placebo and the corresponding erenumab dose group only.

$\mathrm{Cl}$ : confidence interval; MMD: monthly migraine days; $\mathrm{N}$ : total number of patients.

lower in a relatively small $100 \%$ response subgroup compared to patients who did not achieve $100 \%$ response (Figure 6), likely attributable to lower MMD and MSMD at baseline (Table 1). For the subgroups of patients who did not achieve a response at the $\geq 50 \%, \geq 75 \%$, or $100 \%$ thresholds, reductions in MMD were not greater than the overall erenumab-treated population.

\section{Discussion}

In this post hoc analysis, erenumab showed greater efficacy in the response $(\geq 50 \%, \geq 75 \%$, and $100 \%$ reduction from baseline in MMD) subgroups than in the overall-treated population. Both the spectrum of response across all patients and the overall greater reduction in MMD among patients treated with erenumab versus placebo are evident from the waterfall plots. The odds of achieving a response at any threshold was greater in patients treated with erenumab than placebo, and the odds increased for higher thresholds of response. These data complement the results of the primary endpoint, change in MMD at month 3, and underscore the greater magnitude of efficacy realized by patients who achieve $50 \%$ or greater response. Although these analyses focus on efficacy at month 3, significantly greater odds of achieving $\geq 50 \%$ and $\geq 75 \%$ reduction in MMD with both erenumab doses compared with placebo were evident from month 1 (week 4) onwards. Not all patients in the study experienced MMD reduction, and in fact $\sim 20 \%$ of patients across treatment groups did not demonstrate any improvement (change in MMD from baseline $\geq 0$ ), a result that is consistent with other placebo-controlled migraine prevention trials. A challenge for future research is to identify predictors of response/nonresponse to a given preventive treatment.

In clinical trials, treatment effect is assessed by measuring differences in the overall study population based on treatment received. However, response to treatment varies, and not all patients experience the same level of benefit. Hence, an exploratory analysis to better understand the spectrum of treatment benefit among patients, assessing efficacy of erenumab based on different thresholds of response was performed. In this analysis, across all outcome measures at the $\geq 50 \%$ threshold, the magnitude of change was substantially better in responders than in the overall erenumab-treated population and in patients who did not achieve $a \geq 50 \%$ response. This highlights the rather wide spectrum of response to treatment and emphasises that mean results for an overall study population will underestimate the actual benefit achieved in the subgroup of patients who are deemed responders (e.g. using $50 \%$ as a commonly applied response threshold). Similarly, at the $\geq 75 \%$ and $100 \%$ thresholds, a greater effect was observed at both doses 
Table I. Baseline characteristics of response subgroups.

\begin{tabular}{|c|c|c|c|c|c|c|}
\hline \multirow[b]{3}{*}{ Threshold of response } & \multicolumn{3}{|c|}{ Erenumab $70 \mathrm{mg}$} & \multicolumn{3}{|c|}{ Erenumab $140 \mathrm{mg}$} \\
\hline & \multicolumn{2}{|c|}{ Response at given threshold } & \multirow[b]{2}{*}{ Overall population } & \multicolumn{2}{|c|}{ Response at given threshold } & \multirow[b]{2}{*}{ Overall population } \\
\hline & Yes* & No* & & Yes* & No* & \\
\hline \multicolumn{7}{|l|}{$\geq 50 \%$} \\
\hline $\mathrm{N}$ & 75 & 103 & 178 & 77 & 105 & 182 \\
\hline MMD & I7.I (3.4) & $18.6(4.7)$ & $18.0(4.3)$ & I7.0 (4.9) & $18.4(4.5)$ & $17.8(4.7)$ \\
\hline MSMD & $7.5(7.3)$ & $9.9(7.1)$ & $8.9(7.2)$ & $9.3(6.7)$ & $10.3(7.2)$ & $9.9(7.0)$ \\
\hline HIT-6 score & $63.4(5.2)$ & $63.4(4.7)$ & $63.4(4.9)$ & $62.8(6.1)$ & $62.4(5.3)$ & $62.6(5.6)$ \\
\hline MIDAS total score & $58.7(46.1)$ & $67.8(40.5)$ & $64.0(43.0)$ & $54.3(47.9)$ & $65.4(54.5)$ & $60.7(52.0)$ \\
\hline \multicolumn{7}{|l|}{$\geq 75 \%$} \\
\hline $\mathrm{N}$ & 32 & 146 & 178 & 39 & 143 & 182 \\
\hline MMD & I5.8 (3.5) & $18.4(4.3)$ & $18.0(4.3)$ & $17.3(5.2)$ & $17.9(4.6)$ & $17.8(4.7)$ \\
\hline MSMD & $5.0(6.3)$ & $9.8(7.2)$ & $8.9(7.2)$ & $9.8(6.9)$ & $9.9(7.0)$ & $9.9(7.0)$ \\
\hline HIT-6 score & $62.4(5.7)$ & $63.6(4.7)$ & $63.4(4.9)$ & $63.0(6.7)$ & $62.5(5.3)$ & $62.6(5.6)$ \\
\hline MIDAS total score & $53.5(39.2)$ & $66.3(43.6)$ & $64.0(43.0)$ & $48.5(47.0)$ & $63.9(52.9)$ & $60.7(52.0)$ \\
\hline \multicolumn{7}{|l|}{$100 \%$} \\
\hline $\mathrm{N}$ & 8 & 170 & 178 & 5 & 177 & 182 \\
\hline MMD & | $4.5(3.0)$ & |8.| (4.3) & $18.0(4.3)$ & $14.9(2.2)$ & $17.9(4.7)$ & $17.8(4.7)$ \\
\hline MSMD & $2.5(3.6)$ & $9.2(7.2)$ & $8.9(7.2)$ & $4.7(4.5)$ & $10.0(7.0)$ & $9.9(7.0)$ \\
\hline HIT-6 score & $62.4(8.8)$ & $63.4(4.7)$ & $63.4(4.9)$ & $64.8(4.6)$ & $62.5(5.7)$ & $62.6(5.6)$ \\
\hline MIDAS total score & $68.3(44.5)$ & $63.8(43.1)$ & $64.0(43.0)$ & $48.0(14.2)$ & 6I.I (52.6) & $60.7(52.0)$ \\
\hline
\end{tabular}

Data are mean (SD) unless indicated; please refer to Tepper et al. 2017 (I3) for complete demographics and baseline characteristics of the double-blind treatment phase.

*Yes or No indicates response/no response at given threshold. Overall population represents all erenumab-treated population at respective doses. HIT-6: Headache Impact Test (higher score indicates worse outcomes); MIDAS: Migraine Disability Assessment (higher score indicates worse outcomes); MMD: monthly migraine days; MSMD: migraine-specific medication treatment days; N: total number of patients; SD: standard deviation.

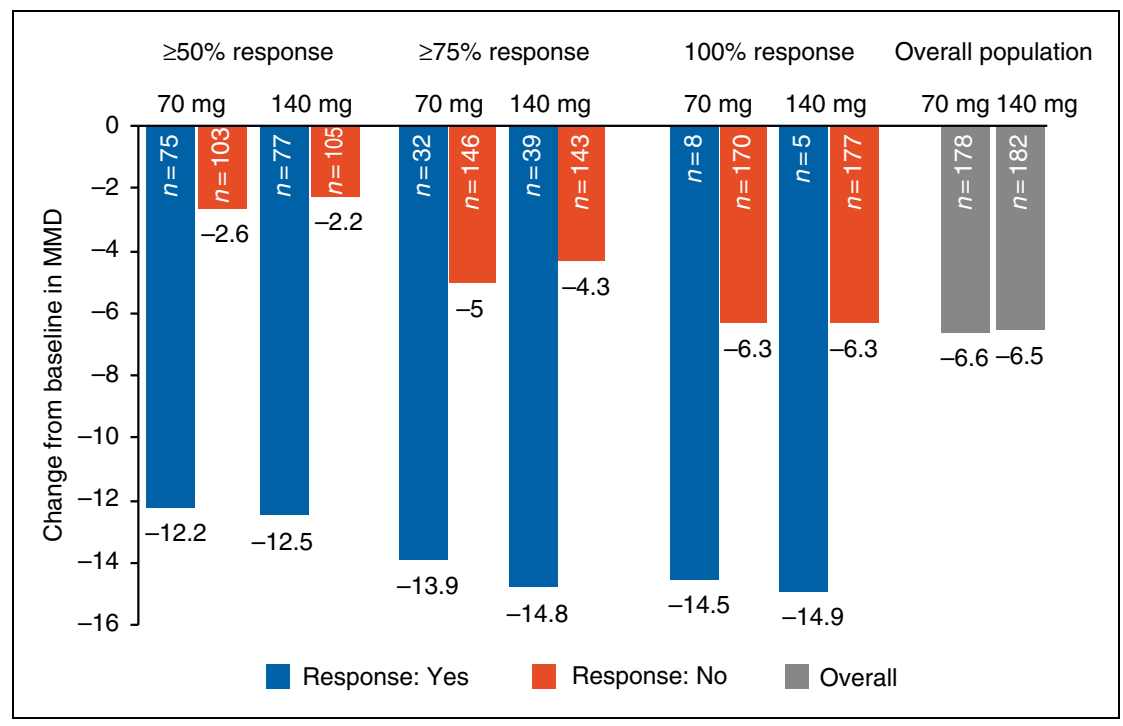

Figure 5. Mean change in MMD from baseline to month 3.

Overall population represents all erenumab-treated population at respective doses.

MMD: monthly migraine days; $n$ : number of patients. 


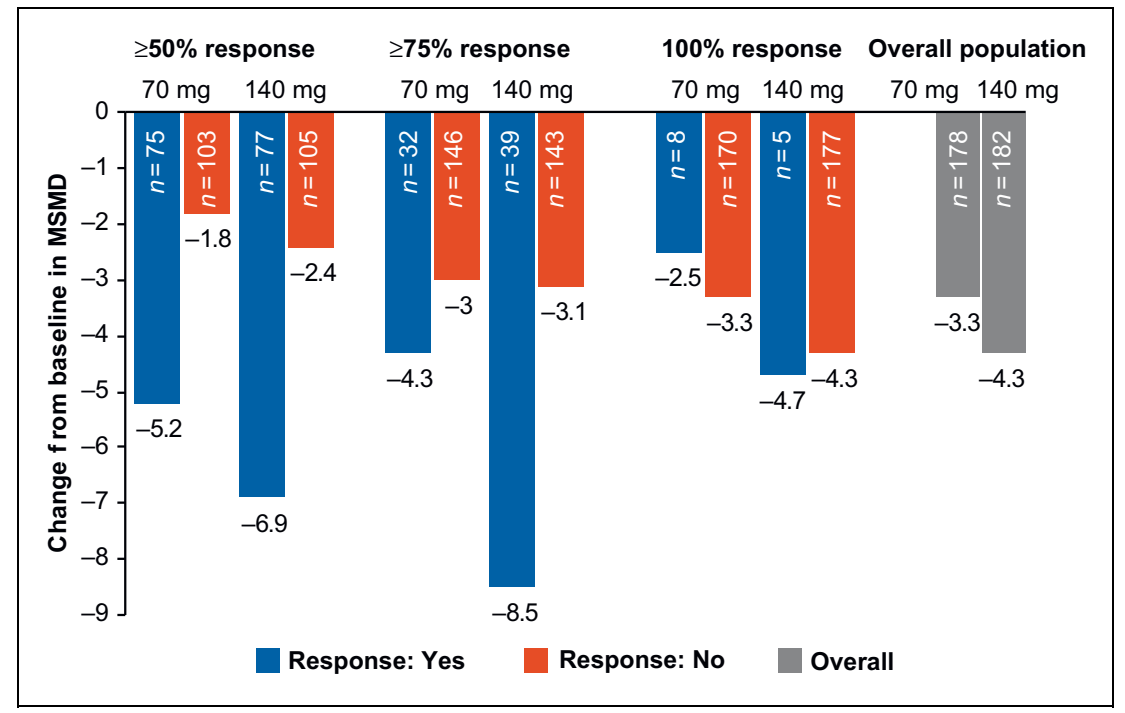

Figure 6. Mean change in MSMD from baseline to month 3.

Overall population represents all erenumab-treated population at respective doses. MSMD: migraine-specific medication treatment days; n: number of patients.

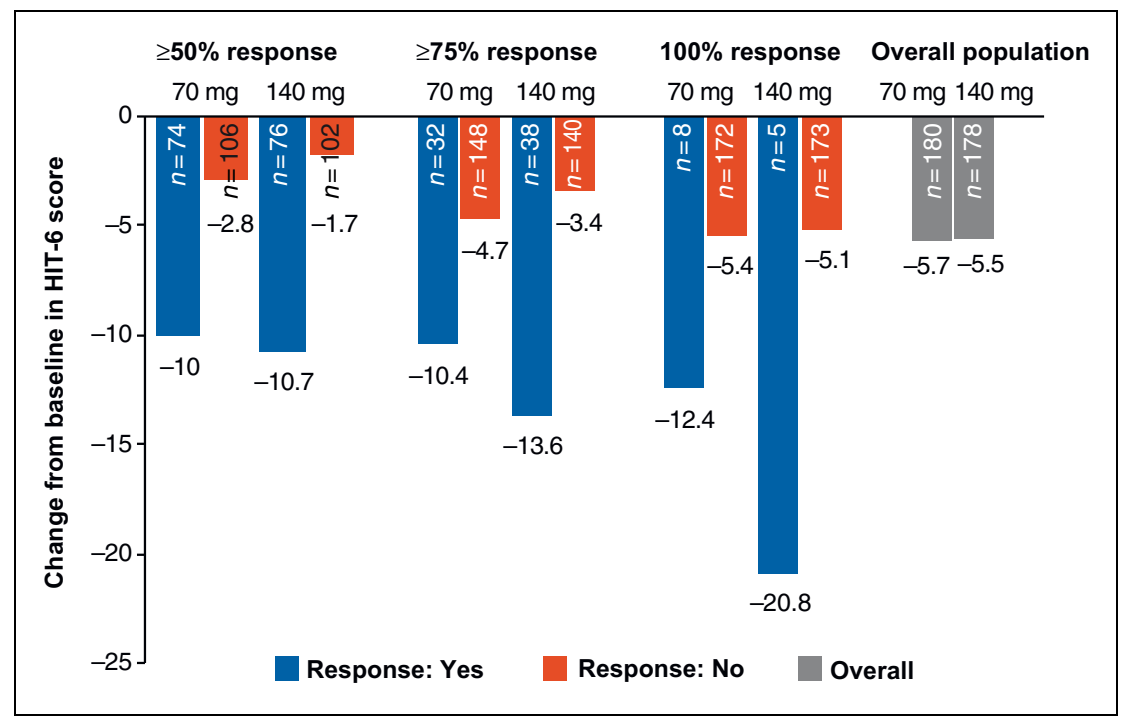

Figure 7. Mean change in HIT-6 from baseline to month 3.

Overall population represents all erenumab-treated population at respective doses.

HIT-6: Headache Impact Test, n: number of patients.

across all outcome measures in responders compared with the overall erenumab-treated population. In an additional analysis performed in subgroups of $\mathrm{CM}$ patients with prior prophylactic treatment failure $(0$, $\geq 1, \geq 2$ and $\geq 3$ ) and medication overuse, erenumab $70 \mathrm{mg}$ and $140 \mathrm{mg}$ demonstrated greater efficacy than placebo $(16,17)$. These results support that patients who respond to erenumab treatment based on the traditional MMD-related responder definition can show substantial treatment benefits on other clinical and functional outcomes. In clinical practice, patients achieving a sufficient response to treatment are more likely to continue the treatment. Hence, these findings are important from a clinical practice perspective as they describe the actual benefits among patients classified as responding to erenumab treatment.

A slight imbalance in baseline characteristics was observed, with lower baseline MMD and MSMD in 


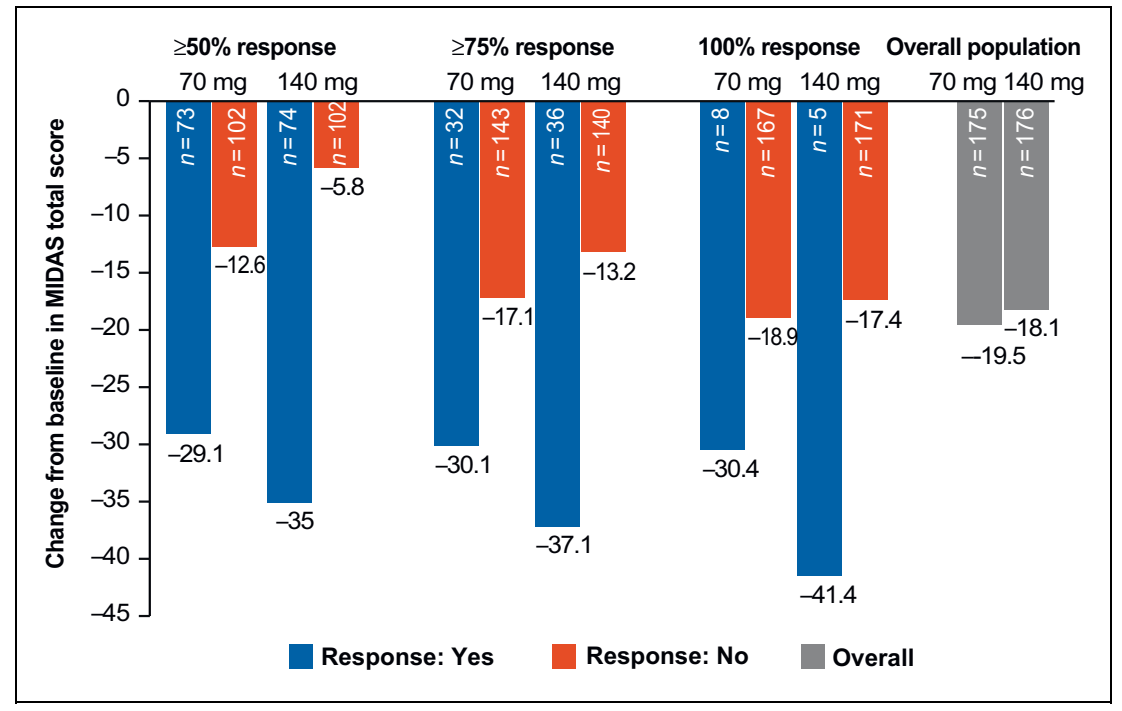

Figure 8. Mean change in MIDAS total score.

Overall population represents all erenumab-treated population at respective doses. MIDAS: Migraine Disability Assessment; n: number of patients.

patients achieving a response versus those not achieving a response at a given threshold. These differences may be attributable, at least in part, to the relatively small number of patients in the responder subgroups at the higher thresholds, particularly at the $100 \%$ threshold. Intuitively, achievement of a higher-level response would appear to be more likely in patients with a lower frequency of events, and this is indeed reflected in the baseline MMD for responders versus those who did not achieve response in both dose groups, with larger differences in baseline MMD at the $\geq 75 \%$ and $100 \%$ thresholds (Table 1). Similarly, baseline MSMD were generally lower in the responder subgroups, again suggesting that lower migraine frequency/severity in a $\mathrm{CM}$ population may be associated with a greater likelihood of a high-level response. Interestingly, baseline HIT-6 scores, a measure of the impact of headache on patients' QoL, was comparable in the subgroups achieving/not achieving responses at a given threshold. Baseline MIDAS scores (a measure of headache-related disability) were higher among patients not achieving a response (more clearly seen at the $\geq 50 \%$ and $\geq 75 \%$ thresholds), suggesting that patients who were most disabled by their migraines, which may be linked to greater frequency of events, may be less likely to achieve a high level of response.

Achievement of $\geq 50 \%$ response is a generally accepted threshold in clinical trials and in clinical practice $(14,15)$. The threshold of response that is deemed clinically meaningful, in terms of relative reduction in migraine days, is likely to be dependent on individual patient characteristics, such as baseline frequency, and likewise a clinically meaningful treatment effect may also depend on other outcomes, such as improvement in pain severity and reduced impact of migraine on functioning $(14,15)$. Furthermore, the response thresholds used are arbitrary and dichotomous, and patients not achieving a given level of response may still experience a treatment benefit. Although there is a clear spectrum of response to treatment, currently, identification of predictors of response represents an important scientific gap. In this analysis, the response rate was assessed over 3 months; future studies should investigate responses over the longer term. This study included patients with medication overuse, history of preventive treatment failure, and comorbidities such as anxiety and depression. However, patients with daily headache, cluster headache, hemiplegic migraine and other comorbidities (e.g. fibromyalgia and poorly controlled hypertension) were excluded from this study and may limit the generalizability of the results to broader populations.

In conclusion, patients treated with erenumab had greater MMD response rates versus placebo at month 3 , regardless of the threshold used to define response. Patients achieving $\geq 50 \%, \geq 75 \%$, and $100 \%$ responses based on change in MMD showed substantially greater reductions not only in migraine days, but also in the use of migraine-specific medication, headache impact, and disability as assessed by the HIT-6 and MIDAS scores, compared with the overall erenumab-treated population. These findings may help to provide context for setting realistic patient expectations regarding the spectrum of response that may be attained at the individual patient level during treatment with erenumab, and for those who respond to treatment, the greater therapeutic gain that may be achieved. 


\section{Clinical implications}

- This post-hoc analysis of a double-blind, placebo-controlled study of erenumab in patients with CM was conducted to contextualize the actual treatment benefit in subgroups of patients achieving specific response thresholds ( $\geq 50 \%, \geq 75 \%$, and $100 \%$ reduction in MMD from baseline).

- At month 3, a greater proportion of patients achieved $\geq 50 \%$ and $\geq 75 \%$ reduction in MMD with erenumab than with placebo.

- Compared with the overall erenumab-treated population, greater reductions in MMD and MSMD were observed amongst $\geq 50 \%$ and $\geq 75 \%$ responders versus those who did not achieve these response thresholds.

- These findings provide context for setting realistic expectations regarding actual treatment benefit experienced by patients responding to treatment.

\section{Acknowledgements}

We thank the study investigators and patients for their participation and commitment to this work. The medical writing support was provided by Dinesh $\mathrm{T}$ Makhija, Novartis Healthcare Pvt. Ltd, India.

\section{Contributors}

All authors participated in the study design, implementation, and/or conduct of the study. All authors contributed to the review of the protocol and approved the final manuscript.

\section{Ethical approval and conduct}

The study protocol was reviewed by the Independent Ethics Committee or Institutional Review Board for each center. The study was conducted according to the ethical principles of the Declaration of Helsinki.

\section{Patient information and consent}

Informed written consent was obtained from each subject before conducting any procedures. Additional information on the study was provided verbally by the study investigator or in a written format.

\section{Declaration of conflicting interests}

The authors declared the following potential conflicts of interest with respect to the research, authorship, and/or publication of this article: JLB has received research grants from Allergan, Teva, Amgen, Alder, Biohaven, Zosano, Colucid, and Eli Lilly and Company and has served on advisory boards/lecturer for Eli Lilly and Company, Teva, Amgen, Promius, Supernus, Valeant, and Avanir.

HCD has received honoraria for participation in clinical trials, contribution to advisory boards or oral presentations from: Alder, Allergan, Amgen, Autonomic Technology, Bristol-Myers Squibb, CoLucid, Electrocore, Ipsen, Lilly, Medtronic, MSD, Novartis, Pfizer, Schaper and Brümmer, Teva and Weber \& Weber. Received financial support for research projects by Allergan, Electrocore, MSD and Pfizer. Headache research at the Department of Neurology in Essen is supported by the German Research Council (DFG), the German Ministry of Education and Research (BMBF) and the European Union. He has no ownership interest and does not own stocks of any pharmaceutical company. He serves on the editorial boards of Cephalalgia and Lancet Neurology. He chairs the Clinical Guidelines Committee of the German Society of Neurology and is a member of the Clinical Trials Committee of the IHS.

DD has received consulting fees, and speaking and/or teaching fees from Allergan, Amgen, Biogen Idec, Novartis, Bayer, and Teva.

MCF has served on advisory boards and/or has consulted or received research support from Alder, Allergan, Avani, Dr. Reddy's Laboratories, Eli Lilly, Scion, and Teva.

PJM has received consulting fee from Alder and research support from Amgen, Biohaven, Eli Lilly, and Teva.

PW is an Investigator in clinical trials sponsored by Teva, Amgen, Genentech, Novartis, Allergan, AstraZeneca, Biogen Idec, Ipsen and Lilly, has participated in advisory boards for Teva, Amgen, Avanir, Novartis, Allergan, Supernus and Lilly, and has been on a speaker's bureau for Allergan, Amgen, Avanir, Lilly, Promius, Novartis, and Supernus.

JK, SW and SR are full-time employees of Novartis. SC, FZ, RAL, and DDM are full-time employees of Amgen.

\section{Funding}

The authors disclosed receipt of the following financial support for the research, authorship, and/or publication of this article: The study was funded by Amgen, Thousand Oaks, CA, USA, and Novartis Pharma AG, Basel, Switzerland.

\section{References}

1. Natoli JL, Manack A, Dean B, et al. Global prevalence of chronic migraine: A systematic review. Cephalalgia 2010; 30: 599-609.

2. Headache Classification Committee of the International Headache Society. The International Classification of Headache Disorders, 3rd edition (beta version). Cephalagia 2013; 33: 629-808.

3. Katsarava Z, Buse DC, Manack AN, et al. Defining the differences between episodic migraine and chronic migraine. Curr Pain Headache Rep 2012; 16: 86-92.

4. Lantéri-Minet M, Duru G, Mudge M, et al. Quality of life impairment, disability and economic burden associated with chronic daily headache, focusing on chronic migraine with or without medication overuse: A systematic review. Cephalalgia 2011; 31: 837-850. 
5. Lipton RB, Bigal ME, Diamond M, et al; on behalf of the AMPP Advisory Group. Migraine prevalence, disease burden, and the need for preventive therapy. Neurology 2007; 68: 343-349.

6. Hepp Z, Dodick DW, Varon SF, et al. Adherence to oral migraine-preventive medications among patients with chronic migraine. Cephalalgia 2015; 35: 478-488.

7. Hepp Z, Bloudek LM and Varon SF. Systematic review of migraine prophylaxis adherence and persistence. J Manag Care Pharm 2014; 20: 22-33.

8. Gracia-Naya M, Santos-Lasaosa S, Ríos-Gómez C, et al. Predisposing factors affecting drop-out rates in preventive treatment in a series of patients with migraine. Rev Neurol 2011; 53: 201-208.

9. Hepp Z, Dodick DW, Varon SF, et al. Persistence and switching patterns of oral migraine prophylactic medications among patients with chronic migraine: A retrospective claims analysis. Cephalalgia 2017; 37: 470-485.

10. Shi L, Lehto S, Zhu DX, et al. Pharmacologic characterization of AMG 334, a potent and selective human monoclonal antibody against the calcitonin gene-related peptide receptor. J Pharmacol Exp Ther 2016; 356: 223-231.

11. Amgen Inc. Aimovig ${ }^{\mathrm{TM}}$ United States prescribing information, https://www.accessdata.fda.gov/drugsatfda_docs/ label/2018/761077s000lbl.pdf (2018, accessed $\quad 18$ November 2019).

12. Novartis Pharma GmbH. Aimovig ${ }^{\circledR}$ (INN-erenumab). Summary of product characteristics, https://www.ema. europa.eu/documents/product-information/aimovigepar-product-information_en.pdf (2018, accessed 18 November 2019).

13. Tepper S, Ashina M, Reuter U, et al. Safety and efficacy of erenumab for preventive treatment of chronic migraine: A randomised, double-blind, placebo-controlled phase 2 trial. Lancet Neurol 2017; 16: 425-434.

14. Tfelt-Hansen P, Block G, Dahlöf C, et al. Guidelines for controlled trials of drugs in migraine: Second edition. Cephalalgia 2000; 20: 765-786.

15. Silberstein S, Tfelt-Hansen P, Dodick DW, et al. Guidelines for controlled trials of prophylactic treatment of chronic migraine in adults. Cephalalgia 2008; 28: 484-495.

16. Ashina M, Tepper S, Brandes JL, et al. Efficacy and safety of erenumab (AMG334) in chronic migraine patients with prior preventive treatment failure: A subgroup analysis of a randomized, double-blind, placebocontrolled study. Cephalalgia 2018; 38: 1611-1621.

17. Tepper SJ, Diener HC, Ashina M, et al. Erenumab in chronic migraine with medication overuse: Subgroup analysis of a randomized trial. Neurology 2019; 92: e2309-e2320. 


\section{DuEPublico}

Duisburg-Essen Publications online
DE UISSBNU R G

Offen im Denken

Ub $\begin{aligned} & \text { universitäts } \\ & \text { bibliothek }\end{aligned}$

This text is made available via DuEPublico, the institutional repository of the University of Duisburg-Essen. This version may eventually differ from another version distributed by a commercial publisher.

DOI: $\quad 10.1177 / 0333102419894559$

URN: urn:nbn:de:hbz:464-20210511-145142-5

This publication is with permission of the rights owner freely accessible due to an Alliance licence and a national licence (funded by the DFG, German Research Foundation) respectively.

(C) International Headache Society 2019. All rights reserved. 